\title{
氧化硼对聚磷酸钲纤维力学和降解性能的影响
}

\author{
强小虎，李涁涁，黄大建，周松毅 \\ (兰州交通大学 材料科学与工程学院, 兰州 730070)
}

摘 要: 采用熔融拉丝法制备聚磷酸钻纤维(Calcium Polyphosphate Fibers, CPPF)。探究不同质量分数的 $\mathrm{B}_{2} \mathrm{O}_{3}$ 对 CPPF 的降解性能和力学性能的影响。利用傅立叶红外光谱仪、扫描电子显微镜(SEM)等对材料的结构及其性能进 行表征。研究表明: 随着 $\mathrm{B}_{2} \mathrm{O}_{3}$ 质量分数的增加, CPPF 的拉伸强度和模量有了明显的提高, 但纤维表面变得粗粘; 同 时 $\mathrm{CPPF}$ 的阻降性能随着 $\mathrm{B}_{2} \mathrm{O}_{3}$ 含量的升高而提高, 且降解后的纤维表面的裂纹减少。 $\mathrm{B}_{2} \mathrm{O}_{3}$ 具有较优的阻降效果, 当 $\mathrm{B}_{2} \mathrm{O}_{3}$ 的添加量为 $9 \%$ 时, 其拉伸强度和模量比未添加的 CPPF 提高了 $146 \%$ 和 $153 \%$; 在降解 $16 \mathrm{~d}$ 后, 其质量损失率 相对于未添加的 CPPF 减少了 $31 \%$ 。

关 键 词: 聚磷酸䥻纤维; $\mathrm{B}_{2} \mathrm{O}_{3}$; 力学性能; 降解性能

中图分类号: TQ174 文献标识码: A

\section{Boron Oxide on Mechanical and Degradation Property of Calcium Polyphosphate Fibers}

\author{
QIANG Xiao-Hu, LI Bin-Bin, HUANG Da-Jian, ZHOU Song-Yi \\ (School of Materrials Science and Engineering, Lanzhou Jiaotong University, Lanzhou 730070, China)
}

\begin{abstract}
Calcium polyphosphate fibers (CPPF) were prepared by melt-drawing method. Effect of $\mathrm{B}_{2} \mathrm{O}_{3}$ with different mass fraction on degradation and mechanical properties of CPPF was studied. The obtained CPPF were characterized by Fourier transform infrared spectroscopy (FT-IR) and scanning electron microscopy (SEM). Results showed that tensile strength and modulus of CPPF were improved obviously with increase of mass fraction of $\mathrm{B}_{2} \mathrm{O}_{3}$. Moreover, the surface morphology of CPPF was influenced by the introducing of $\mathrm{B}_{2} \mathrm{O}_{3}$. In addition, the degradation ratio and cracks on the surface of CPPF decreased with increase of $\mathrm{B}_{2} \mathrm{O}_{3}$ content. Tensile strength and modulus of CPPF with $\mathrm{B}_{2} \mathrm{O}_{3}$ loading of $9 \%$ increased sharply by $146 \%$ and $153 \%$ compared to neat CPPF, respectively. After $16 \mathrm{~d}$, the mass loss ratio of CPPF with $\mathrm{B}_{2} \mathrm{O}_{3}$ loading of $9 \%$ in the degradation process is reduced by $31 \%$ compared to the neat $\mathrm{CPPF}$.
\end{abstract}

Key words: calcium polyphosphate fiber; $\mathrm{B}_{2} \mathrm{O}_{3}$; mechanical property; degradation property

可降解生物材料作为一种新型的医用材料具有 广阔的前景 ${ }^{[1]}$ 。聚磷酸钙(Calcium Polyphosphate, $\mathrm{CPP})$ 是一种以磷酸二氢钻为原料, 通过脱水缩合形 成的一种具有长链结构的无机聚合物 ${ }^{[2-4]}$, 与天然 骨有着类似的化学组成, 具有良好的生物相容性, 是一种有广泛应用前景的可生物降解材料。
目前, CPP 主要的制备形式有支架、颗粒和纤维。 CPP 多孔支架具有较好的力学性能和良好的生物相 容性 ${ }^{[5]}$ 。通过悬浮液热分散复合法, 用硬脂酸作为致 孔剂可制得大孔 $\mathrm{CPP} / \mathrm{CS}$ 复合棒材 ${ }^{[6]}$ 。本实验室之 前通过熔融拉丝的方法制备了聚磷酸钙纤维 $(\mathrm{Cal}-$ cium Polyphosphate Fiber, $\mathrm{CPPF})^{[7]}$, 并以此为增强

收稿日期: 2018-04-20; 收到修改稿日期：2018-05-22

基金项目：国家自然科学基金(31360230); 甘肃省教育厅重点科研项目(2015A-060)

National Natural Science Foundation of China (31360230); Key Research Project of the Department of Education of Gansu Province (2015A-060)

作者简介: 强小虎(1972-), 男, 硕士, 副教授. E-mail: qiangxh@mail.lzjtu.cn 
材料研制了 CPPF/PLA 复合材料, 得到了较好的力 学性能 ${ }^{[8-9]}$; 艾永平等 ${ }^{[10]}$ 则将 CPPF 与不饱和聚酯酰 胺树脂复合, 所得材料的弯曲强度有了很大改善。 因此, 相比 CPP 颗粒, CPPF 有着更好的力学性能和 分散性。但 CPPF 本身作为一种非晶纤维, 其力学 性能并不理想, 而且降解速度较快, 限制了 CPPF 在体内的应用。因此如何提高 CPPF 的力学性能和 阻降性能，成为 CPPF 实际应用急需解决的问题。

目前虽未见 CPPF 改性研究的报道, 但是 CPP 及磷酸盐玻璃的改性报道较为丰富。邱凯等 ${ }^{[11]}$ 研究 了掺锶的 CPP, 发现在锶钙比为 $3: 7$ 时, CPP 的强度 达到最好。Neel 等 ${ }^{[12]}$ 研究了添加不同含量 $\mathrm{Fe}_{2} \mathrm{O}_{3}$ 的 磷酸盐纤维(PGF), 发现直径越大、 $\mathrm{Fe}_{2} \mathrm{O}_{3}$ 含量越高, PGF 的降解速度就越慢。Jonathan 等 ${ }^{[13]}$ 研究发现, 用 少量 $\mathrm{B}_{2} \mathrm{O}_{3}$ 取代 $\mathrm{P}_{2} \mathrm{O}_{5}$ 加入到磷酸盐玻璃中, 即可明 显提高磷酸盐玻璃的阻降性能。因此, 加入氧化物, 如 $\mathrm{B}_{2} \mathrm{O}_{3}$, 能否提高 CPPF 的力学性能和化学稳定性, 有待深入研究。

$\mathrm{B}_{2} \mathrm{O}_{3}$ 作为玻璃形成体, 与磷酸盐结合, 可以形 成嗍磷酸盐玻璃 ${ }^{[14]}$ 。嗍磷酸盐生物活性玻璃与传统 的硅氧网络结构的硅酸盐生物活性玻璃相比, 嗍磷 酸盐具有更高的生物活性，良好的生物相容性 ${ }^{[15]}$ 。 基于本实验室前期工作, 本研究通过添加不同量的 $\mathrm{B}_{2} \mathrm{O}_{3}$ 制备各种 CPPF, 以期提高 CPPF 的力学性能和 阻降性能, 并探究 $\mathrm{B}_{2} \mathrm{O}_{3}$ 对 $\mathrm{CPPF}$ 的降解性能和力学 性能的影响。

\section{1 实验方法}

\section{1 实验材料}

磷酸二氢钲 $\left(\mathrm{Ca}\left(\mathrm{H}_{2} \mathrm{PO}_{4}\right)_{2} \cdot \mathrm{H}_{2} \mathrm{O}\right)$, 分析纯, 由天津 光复精细化工研究所生产; 三氧化二嗍 $\left(\mathrm{B}_{2} \mathrm{O}_{3}\right)$, 分 析纯，由上海建信化工有限公司试剂厂生产。

\section{$1.2 \mathrm{CPPF}$ 的制备}

将 $\mathrm{B}_{2} \mathrm{O}_{3}$ 和 $\mathrm{Ca}\left(\mathrm{H}_{2} \mathrm{PO}_{4}\right)_{2} \cdot \mathrm{H}_{2} \mathrm{O}$ 按质量比为 $0 / 100$ 、 $1 / 99 、 3 / 97 、 5 / 95 、 7 / 93 、 9 / 91$ 分别称取、混合成 $60 \mathrm{~g}$ 混合物, 在粉碎机中粉碎、混合均匀, 加入 $5 \%$ 的蒸 馏水润湿放置 $24 \mathrm{~h}$, 在 $80{ }^{\circ} \mathrm{C}$ 下烘干 $8 \mathrm{~h}$, 移入 $50 \mathrm{~mL}$ 刚玉坩埚中, 高温炉中升温至 $500{ }^{\circ} \mathrm{C}$, 保温 $4 \mathrm{~h}$, 再 升温至 $1200{ }^{\circ} \mathrm{C}$ 保温 $1 \mathrm{~h}$ 。澄清、均化后用拉丝机拉 丝, 得到 $\mathrm{CPPF}$ 。

\section{3 材料表征}

用日本岛津 FTS-3000 傅里叶红外光谱仪(FT-IR) 测定 CPPF 的结构; 采用日本岛津 7000LX 型 X 射 线衍射仪(XRD)对降解前后的样品进行物相分析;
采用日本电子公司的 JSM-5600LV 型扫描电镜(SEM) 对 CPPF 降解前后的表面的显微结构进行观察。

\subsection{CPPF 单丝强度的测试}

参照碳纤维单丝拉伸强度测试标准 $\mathrm{GB} / \mathrm{T}$ 31290-2014, 将单丝 CPPF 放在以 A4 纸作试样祄的 狭槽 $(2.5 \mathrm{~cm} \times 1 \mathrm{~cm})$ 中间, 用胶水将一端固定, 轻轻 沿着狭槽的方向将纤维拉直后用胶水将另一端固定; 采用偏光显微镜测试纤维的单丝直径; 然后将试样 的两端夹在电子万能试验机(E44.304, MST)上, 夹 具间距为 $25 \mathrm{~mm}$, 拉伸速率为 $5 \mathrm{~mm} / \mathrm{min}$, 测试纤维 的拉伸强度和拉伸模量。

\section{5 降解性能测试}

准确称量样品的初始质量 $\left(W_{0}\right)$, 将样品与 $\mathrm{pH}=7.2$ 的磷酸盐缓冲液( $\mathrm{PBS}$ ) 按 $1 \mathrm{~g} / 100 \mathrm{~mL}$ 的比例 放入密封的 $100 \mathrm{~mL}$ 玻璃试管中, 再移入 $(37 \pm 0.5){ }^{\circ} \mathrm{C}$ 的生化培养箱中降解, 每隔 $2 \mathrm{~d}$ 取出一批样品, 用蒸 馏水洗涤 3 次后在 $70{ }^{\circ} \mathrm{C}$ 烘箱中干燥 $8 \mathrm{~h}$, 称重 $\left(W_{1}\right)$ 。 失重率公式如下:

$$
\text { 失重率 }(\%)=\frac{W_{0}-W_{1}}{W_{0}} \times 100 \%
$$

采用 PHS-3C 酸度计测量降解液的 $\mathrm{pH}$ 值, 并绘 制 $\mathrm{pH}$ 值随降解时间的变化曲线。

\section{2 结果与讨论}

\subsection{CPPF 微观结构}

图 1 为不同 $\mathrm{B}_{2} \mathrm{O}_{3}$ 添加量的 CPPF 的微观形貌, 所制得的 CPPF 直径范围为 $15 \sim 25 \mu \mathrm{m}$ 。CPPF 纤维 的表面无禇皱, 但有未溶解的颗粒存在, 并随 $\mathrm{B}_{2} \mathrm{O}_{3}$ 添加量的增加而变多。因此, $\mathrm{B}_{2} \mathrm{O}_{3}$ 的添加量越大, $\mathrm{CPPF}$ 的表面光滑度越差。 $\mathrm{B}_{2} \mathrm{O}_{3}$ 含量为 $10 \%$ 时, 由 于未融或析晶物质过多, 导致无法形成纤维。
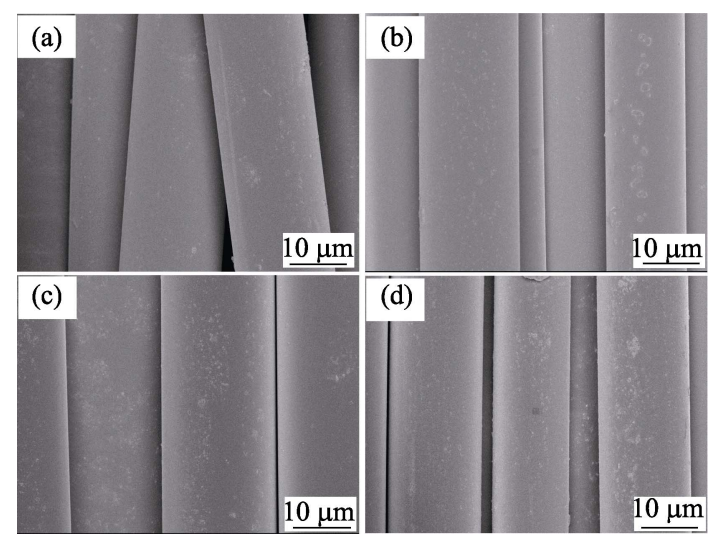

图 1 不同 $\mathrm{B}_{2} \mathrm{O}_{3}$ 添加量的 CPPF 的 SEM 照片

Fig. 1 SEM images of CPPF with different content of $\mathrm{B}_{2} \mathrm{O}_{3}$ (a) CPPF-0; (b) CPPF-1; (c) CPPF-5; (d) CPPF-9 


\section{2 红外分析}

对不同的 CPPF 进行红外分析(图 2), 1271、 1091、902、779 和 $541 \mathrm{~cm}^{-1}$ 处分别出现了明显的吸 收峰, 分别对应于 $\mathrm{P}=\mathrm{O}$ 的伸缩振动峰、 $\mathrm{O}-\mathrm{P}-\mathrm{O}$ 的吸 收振动峰、 $\mathrm{P}-\mathrm{O}-\mathrm{P}$ 的不对称伸缩振动吸收峰、P-O-P 的对称伸缩振动峰, 以及 $\left[\mathrm{PO}_{4}\right]^{3-}$ 的吸收峰 ${ }^{[16]}$ 。这些 特征峰的存在, 证明合成的材料为 $\mathrm{CPP}^{[17]}$ 。而在 $857 \mathrm{~cm}^{-1}$ 处是玻璃中嗍氧四面体 $\left[\mathrm{BO}_{4}\right]$ 的 $\mathrm{B}-\mathrm{O}$ 键的 特征吸收峰 ${ }^{[18]}$ 。因此, 本实验成功地将嗍元素引入 CPPF 的体系中。

\subsection{CPPF 的力学性能}

纤维单丝的拉伸强度和模量是评价纤维力学性 能常用方法。随着 $\mathrm{B}_{2} \mathrm{O}_{3}$ 含量的增加, CPPF 的拉伸 强度和拉伸模量都呈现逐渐增大的趋势(图 3)。在添 加量达到 $9 \%$ 时, 拉伸强度和拉伸模量分别为 1233.9 MPa 和 $202.3 \mathrm{GPa}$ 。比未添加 $\mathrm{B}_{2} \mathrm{O}_{3}$ 的 $\mathrm{CPPF}$, 二者分别提高了近 $146 \%$ 和 $153 \%$ 。可见, $\mathrm{B}_{2} \mathrm{O}_{3}$ 的加 入对 CPPF 的拉伸强度和拉伸模量有着显著的提 高。目前, 在玻璃纤维的直径和成型工艺相同的情 况下, 纤维的理论强度由玻璃的网络结构特性所决 定, 纤维的实际强度受到成型工艺、纤维表面的微

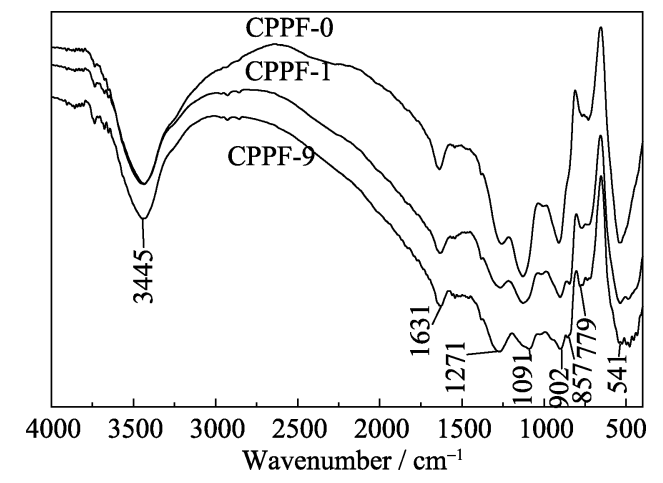

图 2 不同 $\mathrm{B}_{2} \mathrm{O}_{3}$ 添加量的 CPPF 的 FT-IR 图谱

Fig. 2 FT-IR spectra of CPPF with different content of $\mathrm{B}_{2} \mathrm{O}_{3}$

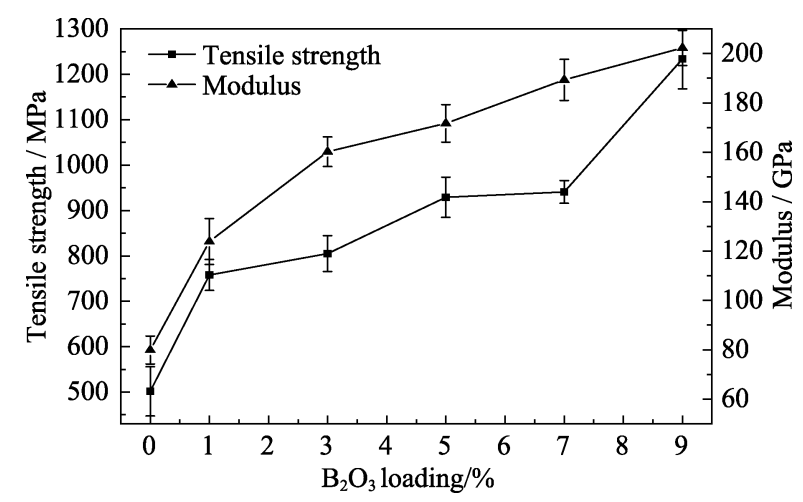

图 $3 \mathrm{~B}_{2} \mathrm{O}_{3}$ 添加量对 CPPF 力学性能的影响

Fig. 3 Effect of $\mathrm{B}_{2} \mathrm{O}_{3}$ loadings on the mechanical properties of CPPF
裂纹、气泡、成分的均匀性等因素的影响，但是纤 维的结构仍是影响纤维强度的主要因素 ${ }^{[19]}$ 。研究表 明, $\mathrm{B}_{2} \mathrm{O}_{3}$ 的加入会使磷酸盐发生结构的变化。 $\mathrm{B}_{2} \mathrm{O}_{3}$ 的加入使 $\left[\mathrm{PO}_{4}\right]$ 四面体中的 $\mathrm{P}=\mathrm{O}$ 键饱和形成网络结 构, 能够促使 $\left[\mathrm{BO}_{4}\right]$ 和 $\left[\mathrm{BPO}_{4}\right]$ 基团的形成, 并由于 B-O 键的键能较大, 使 CPP 的网络结构更加完整。 相比于直链的 CPP, 网状结构的 CPP 更加稳定 ${ }^{[20]}$, 说明加入 $\mathrm{B}_{2} \mathrm{O}_{3}$ 能够增强 CPPF 的力学性能。

\section{$2.4 \mathrm{CPPF}$ 的降解性能}

\subsection{1 纤维降解后的光学照片分析}

不同 $\mathrm{B}_{2} \mathrm{O}_{3}$ 添加量的 CPPF 在 PBS 中降解 4、8 和 $16 \mathrm{~d}$ 后的光学图片(图 4)。在前 $4 \mathrm{~d}$, 只有未添加 $\mathrm{B}_{2} \mathrm{O}_{3}$ 和添加 $1 \% \mathrm{~B}_{2} \mathrm{O}_{3}$ 的样品的试管底部出现了沉淀 物, 此沉淀物为 CPPF 降解产生的 $\mathrm{Ca}^{2+}$ 与 PBS 中磷 酸根产生的羟基磷灰石沉淀 ${ }^{[21]}$, 这可以直观地表现 $\mathrm{CPPF}$ 的降解。而 $\mathrm{B}_{2} \mathrm{O}_{3}$ 添加量达到 $3 \%$ 以上时, 试管 内未发现沉淀，试管清澈透明。在降解 $8 \mathrm{~d}$ 后, $\mathrm{B}_{2} \mathrm{O}_{3}$ 添加量为 $3 \%$ 的样品的试管内, 出现了悬浮的絮状 沉淀物, 而 $5 \%$ 以上的样品未出现沉淀; 在降解 $16 \mathrm{~d}$ 后, CPPF 的降解情况和 $8 \mathrm{~d}$ 时的类似, 只有添加量 小于 $3 \%$ 的样品才出现沉淀或絮状物。这种现象说 明 $\mathrm{B}_{2} \mathrm{O}_{3}$ 的添加对 CPPF 的降解具有较强的影响, $\mathrm{B}_{2} \mathrm{O}_{3}$ 可以明显地阻止 CPPF 的降解, 其原因主要为 $\mathrm{B}_{2} \mathrm{O}_{3}$ 的加入使 CPP 的网络结构更加完整。

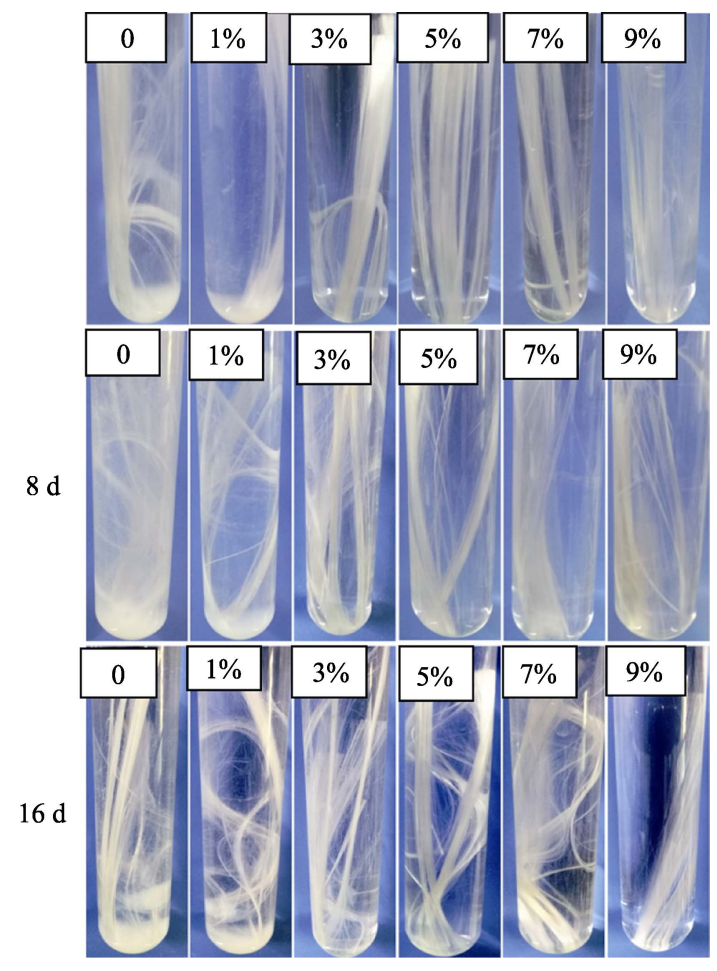

图 4 聚磷酸钲纤维不同时间的降解照片

Fig. 4 Degradation photographs of calcium polyphosphate fiber at different time 


\subsection{2 降解前后的 XRD 分析}

玻璃态结构使远程无序，近程有序，其衍射图 由 1 或 2 个弥散峰组成, $X$ 射线衍射分析在玻璃态中 的应用主要是进行晶化程度的研究、晶相的鉴定等。 CPPF 降解 $16 \mathrm{~d}$ 前后的 XRD 图谱(图 5(A)) 和沉淀物 图谱(图 5(B))显示, CPPF 在降解前后没有出现明显 的衍射峰, 而是呈现出散漫特征的玻璃衍射峰, 说 明 CPPF 在降解前后没有晶相析出; 对照美国标准 卡片 ASTM(09-0432)HA 衍射图谱可知, 图 5(B)衍 射峰位置与标准 HA 衍射图谱的衍射峰位置基本一 致。因此, CPPF 的降解后的沉淀物为羟基磷灰石。 其原因为 $\mathrm{CPPF}$ 降解产生的 $\mathrm{Ca}^{2+}$ 与 $\mathrm{PBS}$ 中磷酸根反 应生成了羟基磷灰石 ${ }^{[21]}$ 。

\subsubsection{CPPF 降解后的扫描电镜照片观察}

CPPF 不同降解时间的扫描电镜照片(图 6)显示, 未降解 CPPF 随着 $\mathrm{B}_{2} \mathrm{O}_{3}$ 含量的增加, 其表面的颗粒 逐渐增多、变大。这是由于玻璃液析晶后残留在纤

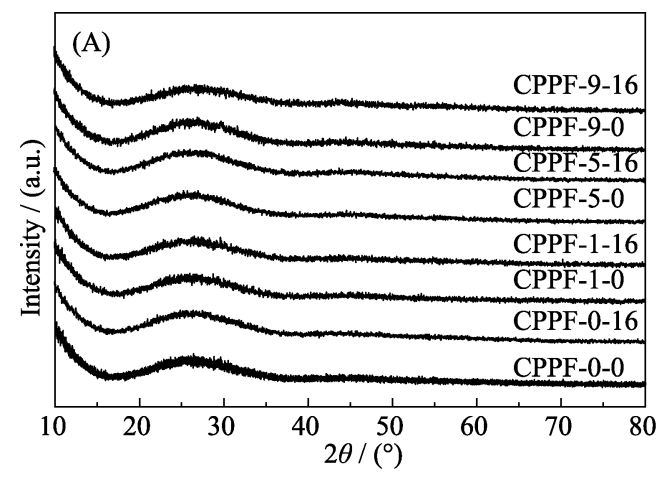

维表面所致; 在降解 $4 \mathrm{~d}$ 后, $\mathrm{B}_{2} \mathrm{O}_{3}$ 添加量为 $0 、 1 \%$ 、 $5 \% 、 9 \%$ 的 CPPF 表面可见纤维开始降解，表面显示 凹凸不平, 有明显的颗粒状; 而随着 $\mathrm{B}_{2} \mathrm{O}_{3}$ 的添加量 的增加, 其表面的颗粒状尺寸减小。降解 $8 \mathrm{~d}$ 后, 纯 CPPF 和添加量为 $1 \%$ 的表面出现了明显的侵蚀坑; 而添加量 5\%和 9\%的 CPPF 表面的颗粒状尺寸变大, 并无明显的侵蚀坑出现。在降解 $16 \mathrm{~d}$ 后, 未添加 $\mathrm{B}_{2} \mathrm{O}_{3}$ 的 CPPF 的表面出现了大面积裂纹; 添加量 $1 \%$ 以上的 CPPF 的表面未出现裂纹。因此, 在降解时 间相同的条件下, 随着 $\mathrm{B}_{2} \mathrm{O}_{3}$ 添加量的增加, $\mathrm{CPPF}$ 表 面的侵蚀坑和裂纹减少。这些现象说明, $\mathrm{B}_{2} \mathrm{O}_{3}$ 的添 加对 CPPF 具有较好的阻降性能。

\subsubsection{CPPF 降解后的 $\mathrm{pH}$ 值变化}

降解期间降解液的 $\mathrm{pH}$ 值得变化趋势(图 7)显示, 6 个样品的 $\mathrm{pH}$ 值在降解期间都呈现出下降趋势。这 是由于 CPPF 在降解液中发生水解反应, 降解产物 中存在磷酸二氢根离子。而 CPPF 降解越快, 降解

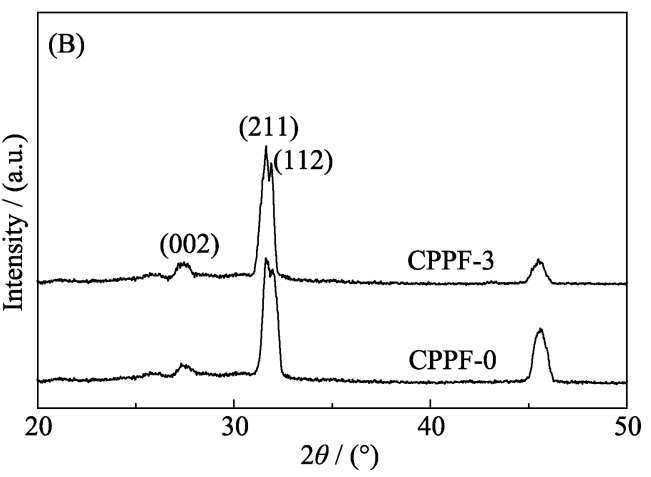

图 5 降解后样品 $(\mathrm{A})$ 和沉淀物的 $\mathrm{XRD}(\mathrm{B})$ 图谱

Fig. 5 XRD spectra of samples (A) and precipitation (B) after degradation

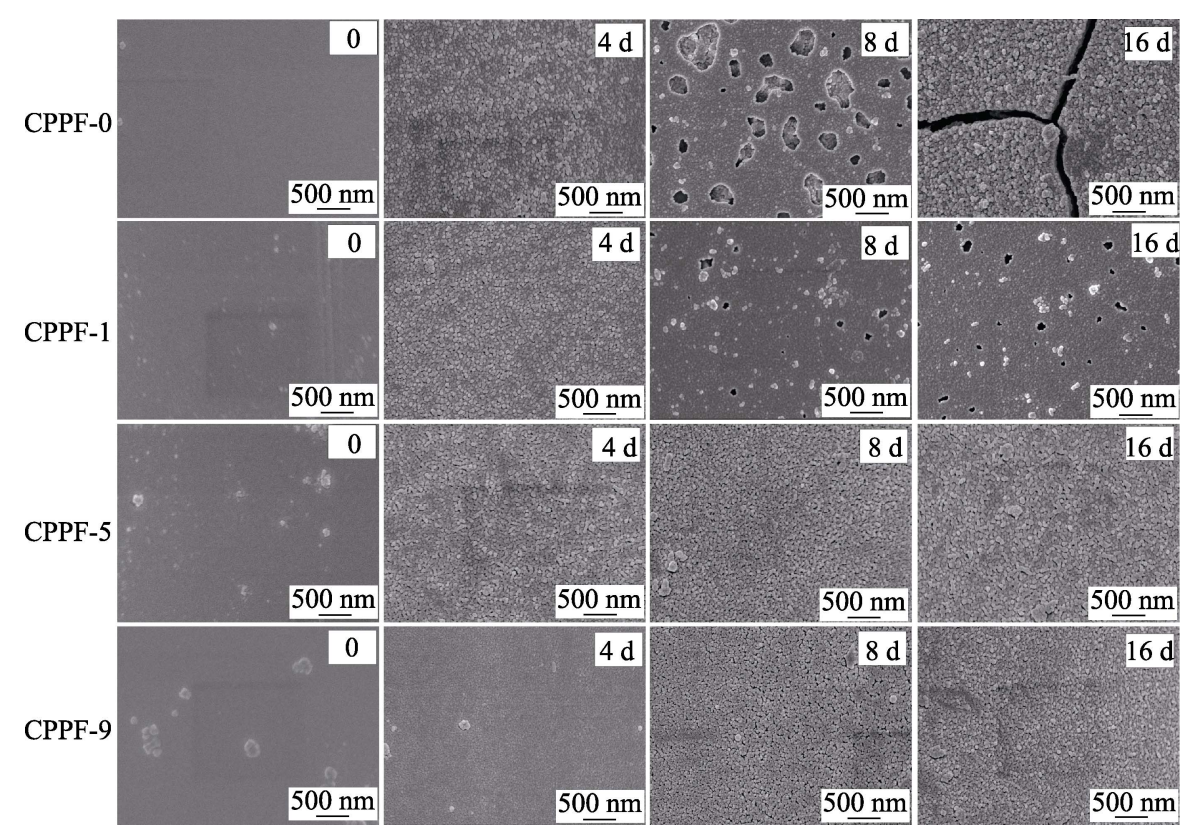

图 6 不同添加量的 CPPF 降解后表面的 SEM 照片

Fig. 6 Surface SEM images of CPPF with different loadings after degradation 
液中的磷酸二氢根离子的浓度则越大，导致降解液 的 $\mathrm{pH}$ 值下降越快 ${ }^{[7]}$ 。纯 $\mathrm{CPPF}$ 样品的 $\mathrm{pH}$ 值下降速 度最快，在第 $16 \mathrm{~d}$ 时 $\mathrm{pH}$ 值达到了 4.15 ; 但添加 7\% 和 $9 \% \mathrm{~B}_{2} \mathrm{O}_{3}$ 的 $\mathrm{CPPF}$ 的 $\mathrm{pH}$ 值，仅前 $2 \mathrm{~d}$ 下降明显， $2 \sim 16 \mathrm{~d}$ 的 $\mathrm{pH}$ 值呈现缓慢下降的趋势。添加量为 $9 \%$ 的样品在降解 $16 \mathrm{~d}$ 后 $\mathrm{pH}$ 值为 6.95 。说明 $\mathrm{B}_{2} \mathrm{O}_{3}$ 的添 加使 CPPF 具有更好的阻降性能。

\subsection{5 纤维降解后的质量变化}

图 8 为不同添加量的 $\mathrm{B}_{2} \mathrm{O}_{3}$ 对 $\mathrm{CPPF}$ 质量变化的 影响曲线图。所有 CPPF 样品降解后的质量比, 随 降解时间的延长而降低, 但随 $\mathrm{B}_{2} \mathrm{O}_{3}$ 添加量的增加, 失重率减小, 即降解速率越慢。添加了 $\mathrm{B}_{2} \mathrm{O}_{3}$ 的 $\mathrm{CPPF}$ 在前 $2 \mathrm{~d}$ 有较大的质量损失, 然后在 2 12 d 的质量 损失趋于平缓。而未掺杂 $\mathrm{B}_{2} \mathrm{O}_{3}$ 的样品的质量损失呈 现线性下降, 在前 $10 \mathrm{~d}$ 的质量损失最大, 失重率达 到 $30 \%$; 在降解 $16 \mathrm{~d}$ 后, 未添加 $\mathrm{B}_{2} \mathrm{O}_{3}$ 样品的失重率 达到 $38 \%$, 而 $\mathrm{B}_{2} \mathrm{O}_{3}$ 添加量为 $9 \%$ 的样品的降解失重 率为 7\%。这说明 $\mathrm{B}_{2} \mathrm{O}_{3}$ 的添加使 CPPF 的质量损失 明显减小, 使 CPPF 的阻降性能增强。这是由于随 着 $\mathrm{B}_{2} \mathrm{O}_{3}$ 的加入, $\mathrm{CPP}$ 由直链结构变为网状结构使得 $\mathrm{CPPF}$ 化学稳定性变得更好。

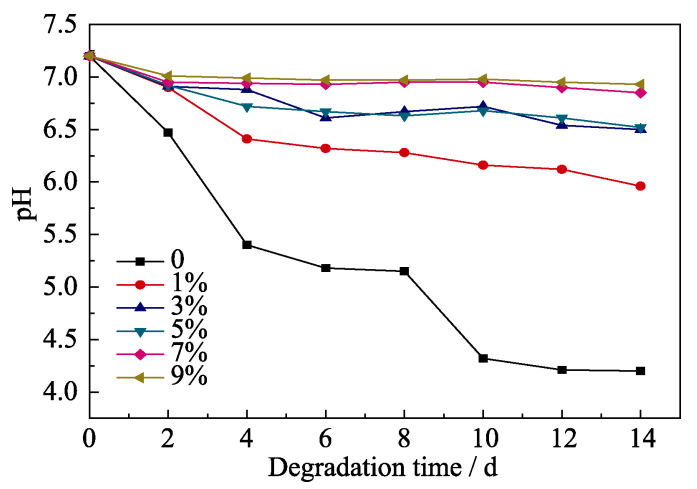

图 7 不同含量的纤维的 $\mathrm{pH}$ 值随降解时间的变化曲线

Fig. $7 \mathrm{pH}$ value in PBS for samples with different loading proportions after degradation for different periods

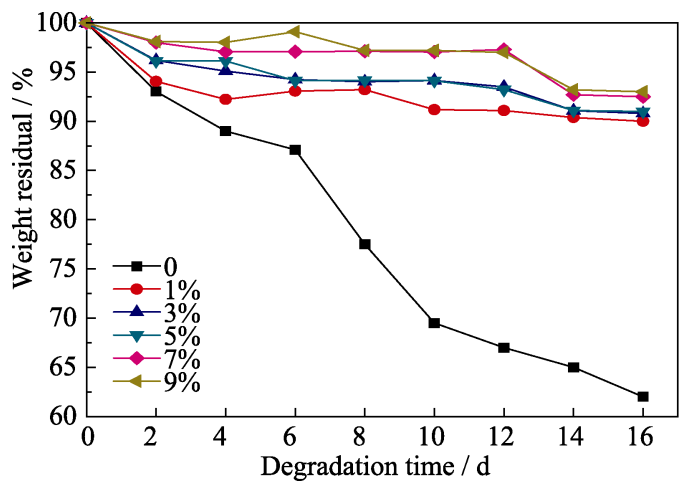

图 8 不同 $\mathrm{B}_{2} \mathrm{O}_{3}$ 含量的 $\mathrm{CPPF}$ 的降解残留率随降解时间的变 化曲线

Fig. 8 Weight residual ratio of calcium polyphosphate fiber with various $\mathrm{B}_{2} \mathrm{O}_{3}$ loadings
从 CPPF 的降解前后的光学照片、显微结构、 $\mathrm{pH}$ 值和质量损失都可以看出, $\mathrm{B}_{2} \mathrm{O}_{3}$ 的加入对 $\mathrm{CPPF}$ 有着明显的阻降作用。

\section{3 结论}

通过在 CPPF 中掺杂 $\mathrm{B}_{2} \mathrm{O}_{3}$, 提高了 CPPF 的力 学性能和化学稳定性。与纯 CPPF 相比, 引入 $9 \%$ $\mathrm{B}_{2} \mathrm{O}_{3}$ 的 CPPF 单丝拉伸强度提高了近 $146 \%$, 拉伸模 量提高了近 $153 \%$ 。而且随着 $\mathrm{B}_{2} \mathrm{O}_{3}$ 添加量的增加, $\mathrm{CPPF}$ 的降解残留率随之增加, 在降解 $16 \mathrm{~d}$ 后, $\mathrm{B}_{2} \mathrm{O}_{3}$ 含量为 $9 \%$ 的 CPPF 降解残留率比纯 CPPF 提高了近 $31 \%$ 。这主要是因为 $\mathrm{B}_{2} \mathrm{O}_{3}$ 将直链状的 $\mathrm{CPP}$ 连接成 网状结构，使其性能更加稳定。因此, $\mathrm{B}_{2} \mathrm{O}_{3}$ 可以提高 CPPF 的力学性能和化学稳定性能。

\section{参考文献:}

[1] DOPPALAPUDI S, JAIN A, KHAN W, et al. Biodegradable polymersan overview. Polym. Adv. Technol, 2014, 25(5): 427-435.

[2] DION A, LANGMAN M, HALL G, et al. Vancomycin release behaviour from amorphous calcium polyphosphate matrices intended for osteomyelitis treatment. Biomaterials, 2005, 26(35): 72-76.

[3] SHANJANI Y, HU Y, PILLIAR R M, et al. Mechanical characteristics of solid-freeform-fabricated porous calcium polyphosphate structures with oriented stacked layers. Acta Biomaterialia, 2011, 7(4): 1788-1796.

[4] BRAY J M, FILIAGGI M J, BOWEN C V, et al. Degradation and drug release in calcium polyphosphate bioceramics: an MRI-based characterization. Acta Biomaterialia, 2012, 8(10): 3821-3831.

[5] PILLIAR R M, KANDEL R A, GRYNPAS M D, et al. Porous calcium polyphosphate as load-bearing bone substitutes: in vivo study. J. Biomed. Mater. Res. B Appl Biomater., 2013, 101B(1): 1-8.

[6] ZHANG Z B, SU Z Q, LI D G, et al. Research on the synthesis and controued degradation of CS / CPP composite for bone repair. Beijing Biomedical Engineering, 2007, 26(2): 144-148.

[7] SHI Z L, CHEN X W, HUANG Y L, et al. Study on mechanical and degradation properties of CPP fibers. Journal of Lanzhou Railway Institute, 1999(5): 62-66.

[8] SHI Z L, ZHUANG Y, QIANG X H, et al. Study on resorbable composite of unidirectional fiber CPP/PLLA for bone fracture fixation. Journal of Mechanical Engineering, 2004, 40(11): 53-57.

[9] QIANG X H, WANG Y P. Degradation of nano-hydroxyapatite/ calcium polyphosphate fiber/polylactide composite. Journal of Clinial Rehabilitative Tissue Engineering Research, 2008, 12(27): 5275-5278.

[10] AI Y P, LIU L J. Fully degradable calcium polyphosphate fiber reinforced unsaturated polyester urea resin used as bone fixed composites. Polymer Materials Science \& Engineering, 2013, 29(10): 109-112, 117.

[11] QIU K, WANG C X, CHEN X. Evaluation of the mechanic properties of the strontium-doped calcium polyphosphate. Chinese Journal of Dental Meterials and Devices, 2005, 14(2): 61-63.

[12] ABOU NEEL E A, AHMED I, BLAKER J J, et al. Effect of iron on the surface, degradation and ion release properties of phosphatebased glass fibres. Acta Biomaterialia, 2005, 1(5): 553-563. 
[13] MASSERA J, SHPOTYUK Y, SABATIER F, et al. Processing and characterization of novel borophosphate glasses and fibers for medical applications. J. Non-Cryst. Solids, 2015, 425: 52-60.

[14] GE M H, LIU W, LI M R, et al. Studies on synthesis and formation of borophosphates under room temperature and ambient pressure. Chinese Journal of Inorganic Chemistry, 2005, 21(6): 826-829.

[15] LIANG W, RAHAMAN M N, DAY D E, et al. Bioactive borate glass scaffold for bone tissue engineering. J. Non-Cryst. Solids, 2008, 354(15/16): 1690-1696.

[16] ZHANG J J, QI Y Y, RUAN D. Influence of different oxide on structure and performance of phosphate glass. Glass, 2014, 41(4): 40-46.

[17] DION A, BERNO B, HALL G, et al. The effect of processing on the structural characteristics of vancomycin-loaded amorphous cal- cium phosphate matrices. Biomaterials, 2005, 26(21): 4486-4494.

[18] ANANTHA P S, HARIHARAN K. Structure and ionic transport studies of sodium borophosphate glassy system. Mater. Chem. Phys., 2005, 89(2): 428-437.

[19] ZU Q. Research on high performance glass fibers. Fiber Glass, 2012(5): 16-23.

[20] YOUSSEF N H, BELKHIRIA M S, VIDEAU J J, et al. Investigation of the physico-chemical properties of calcium borophosphate glasses. effect of the substitution of sodium for calcium. Mater. Lett., 2000, 44(5): 269-274.

[21] SONG W, XU Y T, JIA E P, et al. Effects of doping alkaline metal proportion on degradation and mineral precipitation of calcium polyphosphate. Journal of Sichuan University, 2009, 41(6): 124-129. 